\title{
Comparison of Ultrasonographic Findings with Histo Pathological Findings of Thyroid Nodules - A Two Year Study
}

\author{
Srinivas Metta ${ }^{1}$, Aditya Nutakki ${ }^{2}$ \\ ${ }^{1}$ Associate Professor, Department of Radiology, GSL Medical College Rajamahendravaram, Andhra Pradesh ${ }^{2}$ Sr. Consultant, \\ Chaitanya Medical Center, Maharanipeta, Visakapatnam, Andhra Pradesh, India
}

Corresponding author: Dr. Aditya Nuttaki, Sr. Consultant, Chaitanya Medical Center, Maharanipeta, Visakapatnam, Andhra Pradesh, India

DOI: $10.21276 /$ ijcmsr.2018.3.3.7

How to cite this article: Srinivas Metta, Aditya Nutakki. Comparison of ultrasonographic findings with histo pathological findings of thyroid nodules - a two year study. International Journal of Contemporary Medicine Surgery and Radiology. 2018;3(3):C28-C32.

\section{A B S T R A C T}

Introduction: Thyroid USG plays an important role in demonstrating thyroid anatomy and pathological conditions with remarkable clarity. The frequency of thyroid nodules by sonographic evaluation is $13 \%$ to $67 \%$ based on various literature reports globally. The present study was aimed to evaluate Ultrasonographic morphological features predictive of both benign and malignant thyroid nodules and correlation with FNAC.

Material and methods: A two year prospective study was conducted among patients with thyroid nodules. Ultrasonogram was performed with detailed clinical examination and history taking along with colour Doppler study and differentiated into benign and malignant. FNAC was performed on cases with suspicious malignancy and nodular goitre. Diagnostic statistics viz. Sensitivity, Specificity, PPV, NPV and Accuracy have been computed to find the correlation of Ultrasound and Histopathological diagnosis. $\mathrm{P}$ value: $0.05<\mathrm{P}<0.10$ was considered significant.

Results: 50 cases with $82 \%$ females were included. Majority (54\%) of cases were of solitary nodules and of 50 cases based on USG, $74 \%$ were Benign and $26 \%$ malignant but by FNAC, $78 \%$ were benign and $22 \%$ were malignant. Vascularity, margins, calcification, cystic degeneration and halo were found with significant association with malignancy. (P value $<0.01)$ Sensitivity and specificity of USG for differentiating benign and malignant thyroid nodules were $90.91 \%$ and $92.86 \%$.

Discussion: Our study strongly recommends that features of USG of thyroid nodules are highly suggestive in determining the nodules as benign or malignant and clearly guides in performing FNAC. Our study suggests that Elastography and Colour Doppler USG are newer techniques which are introduced and may be good tools in determining the cases of malignant thyroid nodules. However the techniques are to be standardized.

Key words: Ultrasonogram, Thyroid nodules, Solitary nodule, Colour Doppler.

\section{INTRODUCTION}

Ultrasonography (USG) has become an important diagnostic modality in various disorders of human system. It is an important diagnostic modality in evaluating and differentiating thyroid nodules. Thyroid USG plays an important role in demonstrating thyroid anatomy and pathological conditions with remarkable clarity. Among various other imaging modalities like CT, MRI, USG is least expensive, and is non invasive, easy to perform and has does not involve ionizing radiation and with an additional advantages of real time image acquisition and portability. The frequency of thyroid nodules among general population by clinical examination is $4-7 \%$ whereas by sonographic evaluation is $13 \%$ to $67 \%$ based on various literature reports globally. ${ }^{1}$ The incidence of malignant thyroid nodules is less than $7 \%$. The clinical significance of thyroid nodules is related to exclude thyroid cancer, which is observed in 5-15\% of cases and the causes are multifactorial depending on age, sex and other risk factors. ${ }^{2}$ Various studies conducted have described that Ultrasonographic features like microcalcifications, marked hypoechogenicity, irregular margins and central vascularity are significant characteristics in malignant nodules. These individually may be of limited value but when combined and assessed with combination of FNAC are accurate in predicting malignancy of thyroid nodules. Most of the studies report variability in diagnostic accuracy with overlapping features in USG to differentiate benign and malignant nodules of thyroid. The introduction of new Thyroid stimulating hormone (TSH) assays, widespread application of FNAC and the increasing availability and use of high - resolution USG have facilitated, modified, and improved the management of thyroid pathologies. USG should help in assessing the risk of nodule in determining benign or malignant and help in performance of USGguided FNAC. ${ }^{3}$

The present study was aimed to evaluate Ultrasonographic morphological features predictive of both benign and malignant thyroid nodules. The study also compares the USG features in correlation with FNAC. 


\section{MATERIAL AND METHODS}

The present prospective study was conducted by Department of Radio diagnosis in association with Department of surgery and Department of pathology at a tertiary care Hospital of South India for a period of 2 years from January2015 to December 2017 after approval from the ethical committee of the institute. The details of study were informed to all the patients participating in the study and written consent was obtained. Patients not willing to participate and not willing to give the consent were excluded from the study. Patients of all ages presenting to the OPD of general surgery with Thyroid nodules were referred for ultrasound examination of thyroid. The patient's demographic details, clinical history and signs and symptoms were noted by a senior resident of surgery department. Ultra sonogram of thyroid was performed by a single senior radiologist throughout the study with real time ultrasonographic scanner machine, PHILIPS EnVisor C HD using high frequency linear transducer (3 to $12 \mathrm{MHz}$ ). Curvilinear transducer of lower frequency (2 to 5 $\mathrm{MHz}$ ) was used to measure the size of the thyroid whenever necessary (e.g. in case of marked thyroid enlargement). Colour Doppler Imaging of the thyroid gland was also performed. USG findings were recorded in a written format along with a thermal print of USG images. In case of thyroid nodules features noted were pattern, texture, vascularity, margins, halo, calcifications, colloid or cystic degeneration. Nodules were classified as benign or malignant based on the characteristic features. In cases of multi-nodular goitre, nodule with greatest diameter was considered.

Malignant: Solid nature, hypo-echogenicity, central vascularity, ill-defined margins, micro-calcifications, thick and incomplete halo were taken as features suggestive of malignancy.

Tissue diagnosis with FNAC or postoperative Histo pathological examination was done. FNAC was done under USG guidance and in case of multi-nodular goitre; the nodule with USG features suggestive of malignancy was selected for FNAC.

\section{STATISTICAL ANALYSIS}

Descriptive statistical analysis has been carried out in the present study. Descriptive statistical analysis has been carried out in the present study and categorical measurements in percentage. Chi-square/ Fisher Exact test has been used to find the significance of study parameters on categorical scale between two or more groups. Diagnostic statistics viz. Sensitivity, Specificity, PPV, NPV and Accuracy have been computed to find the correlation of Ultrasound and Histopathological diagnosis. $\mathrm{P}$ value: $0.05<\mathrm{P}<0.10$ was considered significant.

\section{RESULTS}

The present study was conducted for two years and 50 patients were enrolled in the study with 41 (82\%) females and $9(18 \%)$ males. Majority (26\%) were in the age group of 31-40 and $41-50$ years. $54 \%$ of cases presented with solitary nodule, $22 \%$ with two nodules and $24 \%$ with $\geq 3$ nodules. Based on the USG, 37 (74\%) were benign and 13(26\%) were malignant. By Histo pathological examination, 39 were
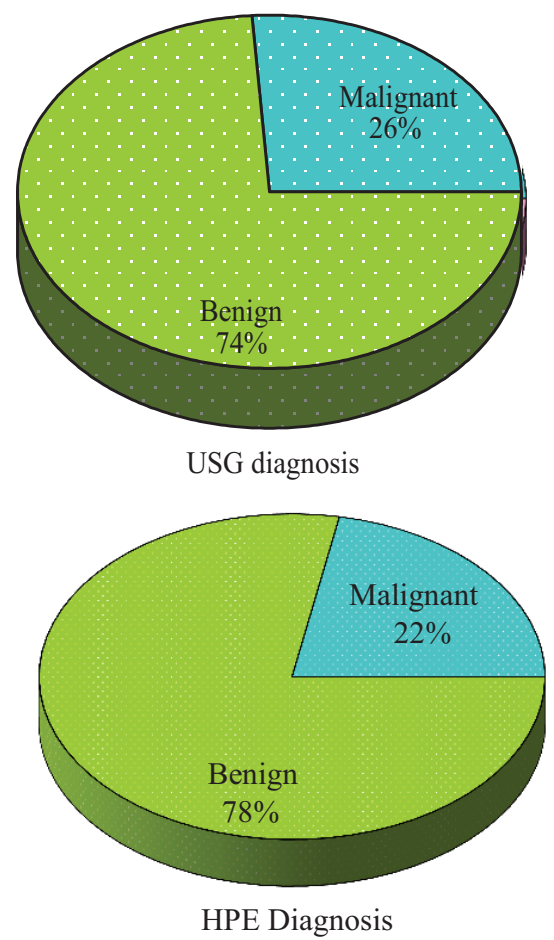

Figure-1: Ultrasound and HPE diagnosis in studied Group.

benign and 11 malignant. Three cases reported as malignant on USG were benign on HPE and one case reported as benign on USG was malignant on Tissue diagnosis. [Fig-1]

\section{USG characteristics of thyroid nodules:}

As described the various characteristics of nodules were studied and noted in a separate sheet. In the present study, $40 \%$ of nodules were solid, $16 \%$ cystic, $40 \%$ solid and cystic and $4 \%$ were cystic with septation. $76 \%$ were heterogenous and $24 \%$ were homogenous. $64 \%$ of the nodules were hypoechogenic, 22\% were isoechogenic and $14 \%$ were hyperechogenic in our study. Nodules with regular margins were observed in $78 \%$ of cases and ill-defined or irregular margins were observed in $22 \%$ of cases. $44 \%$ of the nodules in the study had calcifications with $20 \%$ micro-calcifications, $20 \%$ coarse calcifications and $4 \%$ with egg shell calcifications. $56 \%$ of cases had no calcifications. Halo was observed in $46 \%$ of nodules in the study with thin and complete halo in $38 \%$ and thick and incomplete halo in $8 \%$ of cases. Absence of halo in the nodules was observed in $54 \%$ of cases in the study. Colloid granulations were present in $18 \%$ and cystic degeneration in $50 \%$ of nodules in the present study.

\section{USG characteristics of nodules with FNAC/HPE correlation}

On correlation of USG with HPE, significance ( $\mathrm{P}$ value $<0.01$ ) was found with Vascularity, margins, calcification, cystic degeneration and halo and moderate significance with $\mathrm{P}$ value $<0.05$ was found with texture of the nodule. [Table $-1]$

Features of Benign Nodules: Well defined margins, avascularity and peripheral vascularity, egg shell calcifications, macro calcifications and absence of calcifications and thin and complete halo were observed in all the benign nodules. 


\begin{tabular}{|c|c|c|c|}
\hline \multirow[t]{2}{*}{ Variables } & \multicolumn{2}{|c|}{ HPE diagnosis } & \multirow[t]{2}{*}{$P$ value } \\
\hline & $\begin{array}{l}\text { Benign } \\
(n=39)\end{array}$ & $\begin{array}{c}\text { Malignant } \\
(n=11)\end{array}$ & \\
\hline \multicolumn{4}{|l|}{ Number of nodules } \\
\hline One & $18(46.2 \%)$ & $9(81.8 \%)$ & \multirow[t]{3}{*}{0.102} \\
\hline Two & $9(23.1 \%)$ & $2(18.2 \%)$ & \\
\hline Three and above & $12(30.8 \%)$ & $0(0 \%)$ & \\
\hline \multicolumn{4}{|l|}{ Pattern } \\
\hline Solid & $14(35.9 \%)$ & $6(54.5 \%)$ & \multirow[t]{4}{*}{0.340} \\
\hline Cystic & $8(20.5 \%)$ & $0(0 \%)$ & \\
\hline Solid with cystic component & $15(38.5 \%)$ & $5(45.5 \%)$ & \\
\hline Cyst with septation & $2(5.1 \%)$ & $0(0 \%)$ & \\
\hline \multicolumn{4}{|l|}{ Texture } \\
\hline Heterogeneous & $27(69.2 \%)$ & $11(100 \%)$ & \multirow[t]{2}{*}{$0.046^{*}$} \\
\hline Homogenous & $12(30.8 \%)$ & $0(0 \%)$ & \\
\hline \multicolumn{4}{|l|}{ Echo } \\
\hline Hypo echoic & $24(61.5 \%)$ & $8(72.7 \%)$ & \multirow[t]{3}{*}{0.586} \\
\hline Isoechoic & $8(20.5 \%)$ & $3(27.3 \%)$ & \\
\hline Hyper echoic & $7(17.9 \%)$ & $0(0 \%)$ & \\
\hline \multicolumn{4}{|l|}{ Vascularity } \\
\hline Avascular & $10(25.6 \%)$ & $2(18.2 \%)$ & \multirow[t]{4}{*}{$<0.001^{* *}$} \\
\hline Peripheral vascularity & $15(38.5 \%)$ & $1(9.1 \%)$ & \\
\hline Central vascularity & $1(2.6 \%)$ & $6(54.5 \%)$ & \\
\hline Peripheral and central vascularity & $13(33.3 \%)$ & $2(18.2 \%)$ & \\
\hline \multicolumn{4}{|l|}{ Margins } \\
\hline Well defined & $34(87.2 \%)$ & $5(45.5 \%)$ & \multirow[t]{2}{*}{$0.008^{* *}$} \\
\hline Ill defined & $5(12.8 \%)$ & $6(54.5 \%)$ & \\
\hline \multicolumn{4}{|l|}{ Calcification } \\
\hline Absent calcifications & $26(66.7 \%)$ & $2(18.2 \%)$ & \multirow[t]{4}{*}{$<0.001^{* *}$} \\
\hline Micro calcifications & $1(2.6 \%)$ & $9(81.8 \%)$ & \\
\hline Macro calcifications & $10(25.6 \%)$ & $0(0 \%)$ & \\
\hline Egg shell calcifications & $2(5.1 \%)$ & $0(0 \%)$ & \\
\hline \multicolumn{4}{|l|}{ Halo } \\
\hline Absent & $20(51.3 \%)$ & $7(63.6 \%)$ & $<0.001 * *$ \\
\hline Thin and complete & $19(48.7 \%)$ & $0(0 \%)$ & \\
\hline Thick and incomplete & $0(0 \%)$ & $4(36.4 \%)$ & \\
\hline \multicolumn{4}{|l|}{ Colloid granulation } \\
\hline Absent & $31(79.5 \%)$ & $10(90.9 \%)$ & 0.662 \\
\hline Present & $8(20.5 \%)$ & $1(9.1 \%)$ & \\
\hline \multicolumn{4}{|l|}{ Cystic degeneration } \\
\hline Absent & $15(38.5 \%)$ & $10(90.9 \%)$ & $0.005^{* *}$ \\
\hline Present & $24(61.5 \%)$ & $1(9.1 \%)$ & \\
\hline
\end{tabular}

Features of Malignant nodules: Irregular margins, central vascularity, Micro calcifications, thick and incomplete halo were observed in malignant nodules.

Presence of cystic degeneration was found to be highly suggestive of benignity, though its absence doesn't rule out benignity.

In our study, the sensitivity and specificity of USG for differentiating benign and malignant thyroid nodules were $90.91 \%$ and $92.86 \%$ respectively where as positive predictive value and negative predictive value were $76.92 \%$ and $97.50 \%$ respectively. An accuracy of $92.45 \%$ was obtained. Agreement between US diagnosis and Histopathology was Very good. (Kappa=0.78) (P value
$<0.001)$ Ten patients were diagnosed malignant both by USG and Histopathology (TRUE POSITIVE). Three patients diagnosed as malignant by USG were shown to be benign by histopathology (FALSE POSITIVE).One patient diagnosed as benign sonographically was shown to be malignant histopathologically (FALSE NEGATIVE). Thirty nine patients were diagnosed to be benign both by USG and Histopathology (TRUE NEGATIVE)

\section{DISCUSSION}

High Resolution USG of thyroid plays a very valuable role by identifying thyroid nodules at risk for malignancy depending on certain sonographic features including 
hypoechogenicity, increased vascularity, micro calcifications, irregular margins and the absence of a halo. Ultrasonography guides to determine which nodules require FNAC in cases of small or multiple nodules. In our present study as universally claimed female preponderance was observed with a male to female ratio of $1: 4.5$. In our present study $27 \%$ of females had malignant nodules in comparison with $18.2 \%$ of males with malignant nodules. Inspite of small size of population in our study, the incidence was higher in females as compared with the studies of Sillery JC et $a .^{4}$ Findings of our study were contrary to the findings of Polyzos et al who reported $8 \%$ of malignant thyroid nodules in males as compared to females with $4 \% .{ }^{5}$ In the present study the age variation is very minimal and distributed at a rate of 12 patients under 30 yrs of age, 13 patients are within 31-40 yrs of age, 13 patients were between $41-50$ and 12 patients are more than 50 yrs of age. In our study malignancy is associated with $>40$ years of age and is supported by society of Radiologists in USG consensus conference statement held in 2004, in Washington ${ }^{6}$ and also by Hegedus et al and Keston Jones et al. ${ }^{7,8}$ The incidence of malignant nodules in our study totally was $22 \%$ which is higher than compared to other studies who reported an incidence of $4-12 \%$. Similar incidence of malignant nodules was observed in the study of Kamaljit Kaur et al who reported an incidence of $18 \%$ in her study. ${ }^{9}$ One of the reasons for such a higher incidence of malignancy in our series may be a referral bias as mainly those cases with clinical suspicion is referred for the ultrasonography and for further evaluation like FNAC.

USG plays a key role in assessment and distinguishing benign from malignant nodules based on appearance of sonographic features. Features suggestive of malignancy on USG were hypo echoic pattern, absent / incomplete peripheral halo, irregular margins, micro calcifications, intra nodular vascularity. Features suggestive of benign disease on USG were - Halo sign (transonic uniform rim around the mass), variable echogenicity (hyperechoic/isoechoic/ anechoic), large cystic lesion and absent vascularity or peripheral vascularity. If a solid nodule showed 3 or more features of malignancy with absence of colloid granulations and cystic degeneration was taken as malignant. There were 20 (40.0\%) solid nodules, 8(16.0\%) cystic nodules, 20(40.0\%) solid with cystic component and the rest $2(4.0 \%)$ were cyst with septations. Out of the 20 solid nodules, 6 (54.5\%) were found to be malignant and 5 out of 20 solid nodules with cystic component harboured malignancy. Out of 50 nodules, 32 were hypoechoic and 8 out of these were malignant and 24 benign nodules also showed hypoechoic pattern. A peripheral hypoechoic halo was absent in 7 out of 11 malignant nodules resulting in a sensitivity of $63.6 \%$. Presence of a thick and incomplete halo has a sensitivity of $36.4 \%$ for malignancy. So, the absence of halo and presence of a thick incomplete halo were significant in predicting malignancy. According to Rago et al, the absence of halo has a specificity of $77 \%$ and sensitivity of $67 \%$ in predicting malignancy. ${ }^{10}$ According to USG consensus conference statement, absence of halo has a sensitivity of $17.4-77.5 \%$, specificity of $38.9-85.0 \%$, PPV of $9.3-60.0 \%$ and NPV of $38.9-97.8 \%$.

In our study, micro calcifications were seen in 9 out of
10 malignant cases and in only 1 out of 39 benign cases. Micro calcifications have sensitivity of $81.8 \%$ in predicting malignancy which is in consensus with an article which says, presence of micro calcifications is known to be highly suggestive of malignancy. ${ }^{11}$ Interruption and thickening of peripheral calcifications, and internal echogenicity showed statistical significance in differentiation of benign and malignant nodules in the present study which was similar to reports of Seiberling KA et al. ${ }^{12}$

\section{CONCLUSION}

Our study strongly recommends that features of USG of thyroid nodules are highly suggestive in determining the nodules as benign or malignant and clearly guides in performing FNAC. However based on the features of USG alone Benign or malignant cannot be ruled out.USG definitely would be helpful in selecting better patients for FNAC and in cases where surgery should be indicated with indeterminate cytology. Our study suggests that Elastography and Colour Doppler USG are newer techniques which are introduced and may be good tools in determining the cases of malignant thyroid nodules. However the techniques are to be standardized.

\section{REFERENCES}

1. Tan G H, Gharib H. Thyroid incidentalomas: management approaches to nonpalpable nodules discovered incidentally on thyroid imaging. Ann Intern Med 1997; 126(1): 226-31.

2. Torigian DA, Li G, Alavi A. The role of CT, MR and USG in thyroid imaging PET clinics. 2007; 2 (3):95408.

3. Cappelli C, Castellano M, Pirola I, Cumetti D, Agosti $\mathrm{B}, \mathrm{Gandossi} \mathrm{E}$, et al. The predictive value of ultrasound findings in the management of thyroid nodules. QIM 2007;100 (5):29-35.

4. Sillery JC, Reading CC, Charboneau JW, Henrichsen TL, Hay ID, Mandrekar JN 2010 Thyroid follicular carcinoma: sonographic features of 50 cases. Am J oentgenol 194 (2):44-54.

5. Polyzos SA, Kita M, Avramidis A.Thyriod nodules Stepwise diagnosis and management, Hormones 2007; 6 (4):101-119.

6. Frates $\mathrm{MC}$, Benson $\mathrm{CB}$, Charboneau JW, et al. Management of thyroid nodules detected at US: Society of Radiologists in ultrasound consensus conference statement. Radiology, 2005;237 (6):794-800.

7. Hegedus L, Bonnema SJ, BennedbackFN. Management of simple nodular goiter: current status and future perspectives. Endocr Rev 2003; 24 (4):1-2-132.

8. Frates MC, Benson CB, Doubilet PM, et al.Likehood of thyroid cancer based on sonographic assessment of nodule size and composition. In: Radiological Society of North America Scientific Assembly and Annual Meeting Program. Oak Brook, III: Radiological Society of North America, 2004; (2):395.

9. Kaur K, Sonkhya N, Bapna A.S, Mital P.A comparative study of find needle aspiration cytology, ultrasonography and radionuclide scan in the management of solitary thyroid nodule: A prospective analysis of fifty cases; 
Indian Journal of otolaryngology and Head and neck surgery. 2002; 54 (1):96-101.

10. Rago T, Vitti P, Chiovata L, et al. Role of conventional ultrasonography and color flow-Doppler sonography in predicting malignancy in 'cold' thyroid nodules. Eur J Endocrinol 1998;138 (5):41-46.

11. Minjung Park, MD, Jung Hee Shin, MD, Boo-Kyung Han, MD, Eun Young Ko, MD Sonography of Thyroid Nodules with Peripheral Calcifications. Journal of clinical ultrasound 2009; (4) 324-328.

12. Seiberling KA, Dutra JC, Grant T, Bajramovic S 2004 Role of intrathyroidal calcifications detected on ultrasound as a marker of malignancy. Laryngoscope 114 (1): 1753-1757.

Source of Support: Nil; Conflict of Interest: None

Submitted: 25-06-2018; Accepted: 02-08-2018; Published online: 07-08-2018 